\title{
Improving IRMPD in a Quadrupole Ion Trap
}

\author{
G. Asher Newsome and Gary L. Glish \\ Department of Chemistry, University of North Carolina at Chapel Hill, Chapel Hill, North Carolina, USA
}

A focused laser is used to make infrared multiphoton photodissociation (IRMPD) more efficient in a quadrupole ion trap mass spectrometer. Efficient (up to $100 \%$ ) dissociation at the standard operating pressure of $1 \times 10^{-3}$ Torr can be achieved without any supplemental ion activation and with shorter irradiation times. The axial amplitudes of trapped ion clouds are measured using laser tomography. Laser flux on the ion cloud is increased six times by focusing the laser so that the beam waist approximates the ion cloud size. Unmodified peptide ions from $200 \mathrm{Da}$ to $3 \mathrm{kDa}$ are completely dissociated in $2.5-10 \mathrm{~ms}$ at a bath gas pressure of $3.3 \times 10^{-4}$ Torr and in 3-25 ms at $1.0 \times 10^{-3}$ Torr. Sequential dissociation of product ions is increased by focusing the laser and by operating at an increased bath gas pressure to minimize the size of the ion cloud. (J Am Soc Mass Spectrom 2009, 20, 1127-1131) (C) 2009 Published by Elsevier Inc. on behalf of American Society for Mass Spectrometry

I nfrared multiphoton photodissociation (IRMPD) has been used for tandem mass spectrometry in a quadrupole ion trap mass spectrometer [1-7] and a Fourier transform ion cyclotron resonance mass spectrometer [8-11] as an alternative to collision-induced dissociation (CID). Only one $\mathrm{m} / \mathrm{z}$ ion is selected for excitation in conventional CID, limiting sequential dissociation of product ions into smaller mass product ions. Conversely, in IRMPD all parent and product ions are irradiated simultaneously with a collimated, IR laser providing the capability to generate abundant small mass product ions. IRMPD is typically performed in a quadrupole ion trap mass spectrometer at a smaller low mass cut-off (LMCO) than CID, allowing the observation of a broader $m / z$ range of product ions $[2,4]$, and a higher MS/MS efficiency,

$$
E_{M S / M S}=\frac{\sum_{i} F_{i}}{P_{0}} \times 100 \%
$$

where $E_{M S / M S}$ is MS/MS efficiency, $F_{i}$ is the abundance of each product ion, and $P_{0}$ is the initial abundance of the parent ion before irradiation. IRMPD is performed in a Fourier transform ion cyclotron resonance mass spectrometer to eliminate the need for a CID target gas to be leaked into and pumped out of the ICR cell $[10,11]$.

The sensitivity of a quadrupole ion trap mass spectrometer is optimized with a bath gas pressure around $1.0 \times 10^{-3}$ Torr. Bath gas collisions reduce the kinetic energy of trapped ions and minimize the size of the ion cloud [12]. However, collisions also remove internal energy gained from IR absorption, necessitating longer

Address reprint requests to Professor G. L. Glish, Department of Chemistry, University of North Carolina at Chapel Hill, Chapel Hill, NC 27599-3290, USA. E-mail: glish@unc.edu periods of irradiation for dissociation and decreasing fragmentation efficiency [13],

$$
E_{F}=\frac{\sum_{i} F_{i}}{\sum_{i} F_{i}+P} \times 100 \%
$$

where $E_{F}$ is fragmentation efficiency, $F_{i}$ is the abundance of each product ion, and $\mathrm{P}$ is the abundance of the parent ion remaining after irradiation. IRMPD in a quadrupole ion trap mass spectrometer often is performed at $1 \times 10^{-5}$ to $3 \times 10^{-4}$ Torr to decrease collisional cooling of parent ion internal energy [2-7], [13] and achieve dissociation with shorter irradiation times. However, this reduced bath gas pressure is less effective for trapping ions during injection and reduces sensitivity.

Several methods have been developed to increase IRMPD fragmentation efficiency by combining IRMPD with another form of ion activation and/or decreasing collisional cooling. Ions have been dissociated in a quadrupole ion trap mass spectrometer at $1 \times 10^{-3}$ Torr with irradiation times comparable to IRMPD at lower pressures by using thermal assistance [4] or collisional activation before irradiation [14]. Pulsed introduction of the bath gas has also been used to increase trapping efficiency during ion injection, and then the gas is pumped away before irradiation [15]. Covalent attachment of IR-active ligands to increase the rate of absorption has been used to achieve dissociation in shorter irradiation times $[16,17]$. The first example of IRMPD in a quadrupole ion trap mass spectrometer used a multipass optical system to increase the path length of the laser and overlap with the ion cloud [1]. In Fourier transform ion cyclotron resonance mass spectrometers collisional cooling is not an issue, but because of the large magnetron radius of the ions the laser is commonly left unfocused to maximize laser overlap [18];
(C) 2009 Published by Elsevier Inc. on behalf of American Society for Mass Spectrometry. $1044-0305 / 09 / \$ 32.00$

doi:10.1016/j.jasms.2009.02.003
Published online February 10, 2009 Received January 22, 2009 Revised February 4, 2009 Accepted February 4, 2009 
irradiation times are commonly up to one order of magnitude longer than for IRMPD in a quadrupole ion trap mass spectrometer. The work reported here shows that focusing the laser for IRMPD in a quadrupole ion trap mass spectrometer is the most simple and effective means of increasing fragmentation efficiency. Peptide ions are shown here to be completely dissociated in as little as $3 \mathrm{~ms}$ by focusing the laser on the trapped ion cloud at $1.0 \times 10^{-3}$ Torr, at least an order of magnitude shorter than irradiation times previously reported with a quadrupole ion trap mass spectrometer at any bath gas pressure.

\section{Experimental}

All experiments were performed on a modified Finnigan ITMS controlled with ICMS software [19]. Peptides were obtained from Sigma Chemical Co. and used without further purification. Samples were prepared as $25 \mu \mathrm{M}$ solutions in 75:20:5 methanol:water:acetic acid. Base pressure in the quadrupole ion trap mass spectrometer without added bath gas was $2 \times 10^{-5}$ Torr. Helium bath gas was added for a constant pressure of $3 \times 10^{-4}$ Torr to $1 \times 10^{-3}$ Torr. (Measurements were made at $3 \times 10^{-4}$ Torr because this is the pressure just below where significant collisional cooling starts to occur [4].) There are no conductance limits in the electrode assembly so the pressure measured in the vacuum system is the pressure in the quadrupole ion trap mass spectrometer. A nano-electrospray source was used to generate parent ions: $[\mathrm{PD}+\mathrm{H}]^{+}(231 \mathrm{Da}$, $m / z$ 231); [ALILTLVS + $\mathrm{H}^{+}$(830 Da, $m / z$ 830); [bradykinin $+2 \mathrm{H}]^{2+}(1062 \mathrm{Da}, m / z 531)$; and [melittin $\left.+4 \mathrm{H}\right]^{4+}$ (2850 Da, $m / z$ 712). All parent ions were trapped at a $\mathrm{q}_{\mathrm{z}}$ of 0.10 .

A Synrad $50 \mathrm{~W} \mathrm{CO}_{2}$ laser triggered by a TTL pulse from the ITMS electronics is used for IRMPD. The laser beam is passed into the vacuum housing through a ZnSe window by optical elements mounted on translation stages. A ZnSe lens with a focal length of $38.1 \mathrm{~cm}$ can be translated into or out of the laser path. The ring electrode has been modified by drilling a $3.2 \mathrm{~mm}$ hole through the center, perpendicular to the axial direction. The laser beam is passed through the hole and out the opposite side of the ring electrode into a beam dump (the inside of a [1/2]-inch Cajon fitting painted black with Aerodag).

IRMPD fragmentation efficiency measurements are used to center the laser beam on the trapped ion cloud for maximum overlap, with and without the focusing lens. A peptide ion is trapped at $\mathrm{q}_{\mathrm{z}}$ of 0.40 to minimize the axial size of the ion cloud. The laser beam is translated axially until maximum dissociation is observed. The laser beam is translated to either side to confirm the position at the center of the ion cloud, as dissociation is reduced at equal distances from the center. The vertical position of the laser beam is also adjusted for maximum dissociation.

\section{Results and Discussion}

\section{Laser Power and Overlap with the Ion Cloud}

The beam waist of the unfocused and focused laser was measured at the focal length, outside the vacuum system. A razor blade on a precision translation stage was used to measure the beam waist by finding the positions where beam power was attenuated by $7 \%$ and $93 \%$, defined as the beam diameter, and dividing the difference by $\sqrt{2}$ to relate the Gaussian beam profile to a full width at half maximum. The unfocused laser beam waist was measured nine times for an average of $3.63 \pm 0.07 \mathrm{~mm}$, but in IRMPD experiments the laser beam is clipped at the edge of the smaller, $3.2 \mathrm{~mm}$ hole in the ring electrode. The focused laser beam waist was also measured nine times at the focal point for an average of $0.94 \pm 0.04 \mathrm{~mm}$.

The axial amplitudes of trapped ion clouds at a $\mathrm{q}_{\mathrm{z}}$ of 0.10 and helium bath gas pressure of $3.3 \times 10^{-4}$ Torr, reduced from $1.0 \times 10^{-3}$ Torr to prevent significant collisional cooling for IRMPD, were measured with laser tomography [20, 21] (Figure 1). The focused laser was axially translated away from the center of the trapping volume up to $\sim 1 \mathrm{~mm}$ without clipping the edge of the hole in the ring electrode. Overlap with the ion cloud decreases as the focused laser beam is translated axially, and fragmentation efficiency decreases accordingly. The larger $\mathrm{m} / \mathrm{z}$ ion had a smaller measured ion cloud at the same $\mathrm{q}_{\mathrm{z}}$, as predicted [22]. Ion cloud axial amplitudes have also been shown to decrease with increasing bath gas pressure [20]. The profile of $[\mathrm{PD}+\mathrm{H}]^{+}$at $3.3 \times 10^{-4}$ Torr and $\mathrm{a} \mathrm{q}_{\mathrm{z}}$ of 0.10 represents the largest ion cloud in these IRMPD experiments and is larger than the beam waist of the focused

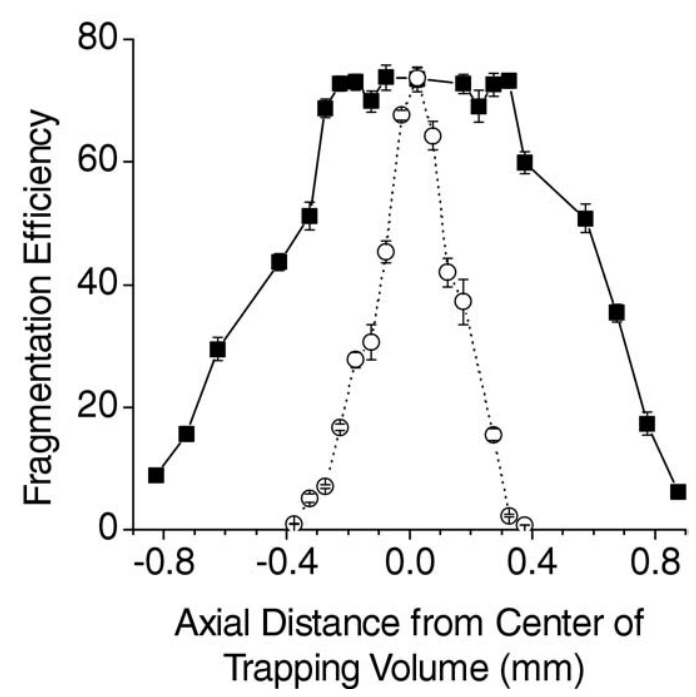

Figure 1. Laser tomography of $[\mathrm{PD}+\mathrm{H}]^{+}$(filled square), $m / z$ 231, and [melittin $+4 \mathrm{H}]^{4+}$ (open circle), $m / z 712$, at a constant helium bath gas pressure of $3.3 \times 10^{-4}$ Torr and a $\mathrm{q}_{\mathrm{z}}$ of $0.10(95 \%$ confidence error bars). Constant irradiation times of $7 \mathrm{~ms}$ for $[\mathrm{PD}+\mathrm{H}]^{+}$and $1.75 \mathrm{~ms}$ for $[\text { melittin }+4 \mathrm{H}]^{4+}$ were used to approximately match peak fragmentation efficiency. 

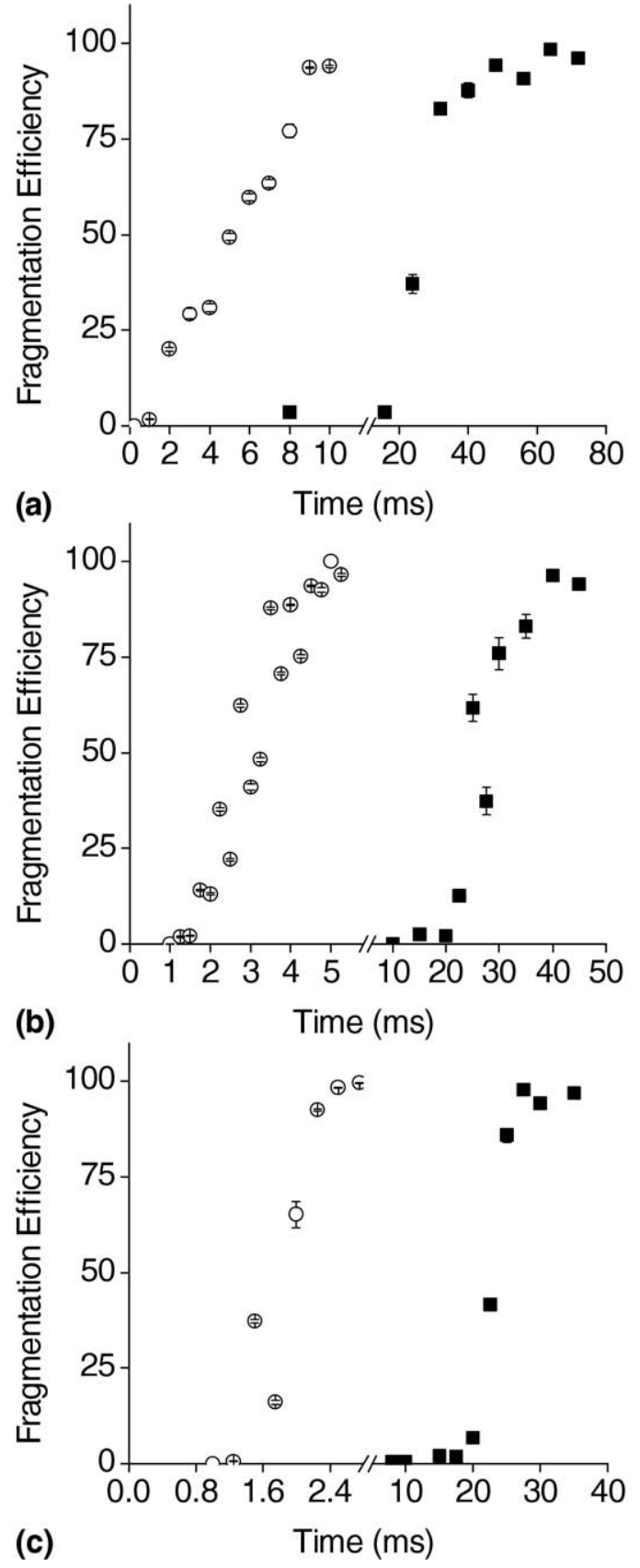

Figure 2. IRMPD at a constant helium bath gas pressure of $3.3 \times$ $10^{-4}$ Torr with the laser unfocused (filled square]) and focused (open circle) of $(\mathbf{a})[\mathrm{PD}+\mathrm{H}]^{+}, m / z 231 ;(\mathbf{b})[\text { bradykinin }+2 \mathrm{H}]^{2+}$, $\mathrm{m} / \mathrm{z} 531$; and (c) [melittin $+4 \mathrm{H}]^{4+} \mathrm{m} / \mathrm{z} 712(95 \%$ confidence error bars).

laser. The unfocused laser irradiates an area up to three times larger than trapped ion clouds, and much of the radiation is not absorbed because the ion cloud does not overlap the whole beam spot. The focused laser beam is smaller than or similar in size to all trapped ion clouds, increasing the overlap of the ion cloud with the laser. Laser flux on the ion cloud is also increased by focusing laser intensity on a smaller area.

The laser beam power was measured at two positions: the center of the ring electrode and $30.5 \mathrm{~cm}$ beyond the center of the ring electrode. Laser power at the center of the trap was measured external to the vacuum housing by passing the laser through a separate, $3.2 \mathrm{~mm}$ aperture equal to the hole in the ring electrode. The power of the unfocused laser was reduced by clipping at the edge of the hole in the ring electrode, and the power of the focused laser was $1.9 \pm$ 0.3 times greater. Taking the focused beam waist and
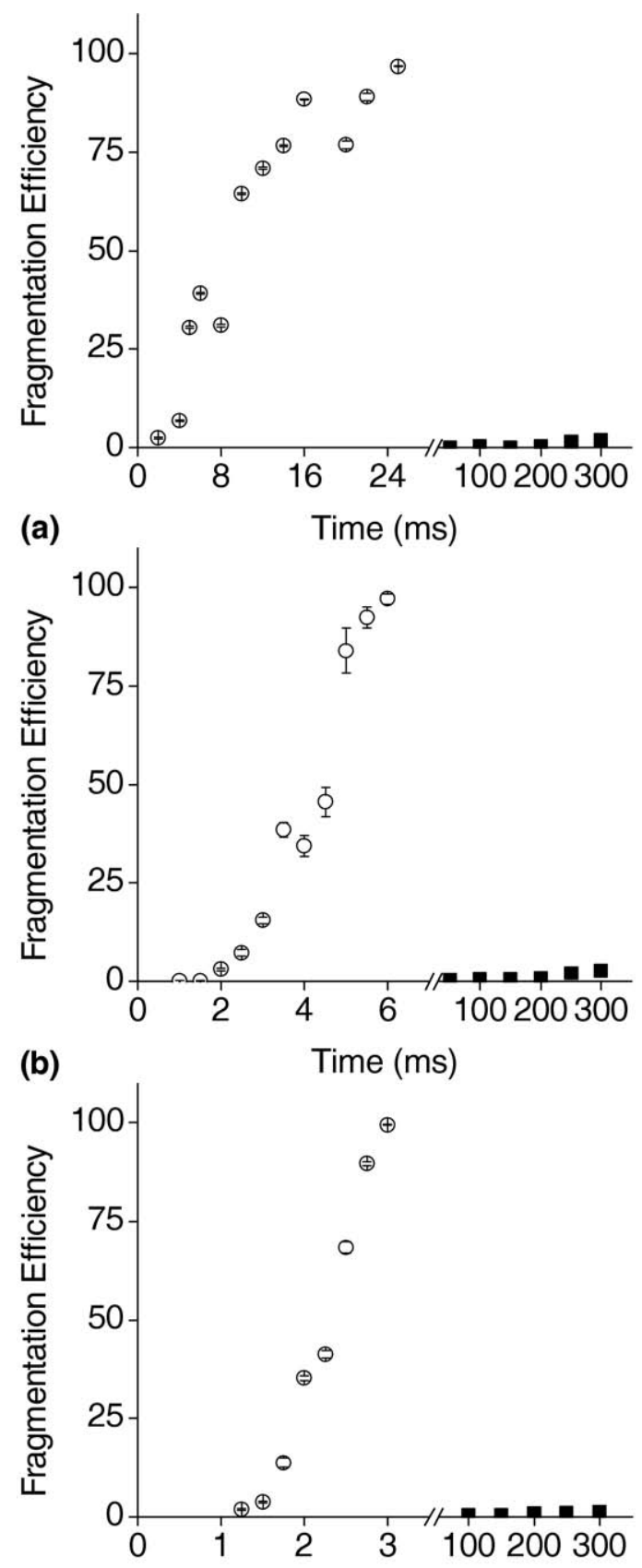

(c) Time (ms)

Figure 3. IRMPD at a constant helium bath gas pressure of $1.0 \times$ $10^{-3}$ Torr with the laser unfocused (filled square]) and focused (open circle) of (a) $[\mathrm{PD}+\mathrm{H}]^{+}, \mathrm{m} / z 231$; (b) [bradykinin $\left.+2 \mathrm{H}\right]^{2+}$, $\mathrm{m} / \mathrm{z} 531$; and (c) [melittin $+4 \mathrm{H}]^{4+} \mathrm{m} / \mathrm{z} 712(95 \%$ confidence error bars). 
Table 1. Product ion abundances from IRMPD of [ALILTLVS $+\mathrm{H}]^{+}$with the laser unfocused at helium bath gas pressure of $3.3 \times$ $10^{-4}$ Torr

\begin{tabular}{ccccccccc}
\hline $\begin{array}{c}\text { Irradiation } \\
\text { time }(\mathrm{ms})\end{array}$ & $\begin{array}{c}\text { \% Fragmentation } \\
\text { efficiency }\end{array}$ & $\begin{array}{c}\% \mathrm{MS} / \mathrm{MS} \\
\text { efficiency }\end{array}$ & $\begin{array}{c}\mathrm{b}_{7}^{+} \\
\text {abundance }\end{array}$ & $\begin{array}{c}\left.\mathrm{b}_{6}-\mathrm{H}_{2} \mathrm{O}\right]^{+} \\
\text {abundance }\end{array}$ & $\begin{array}{c}\mathrm{b}_{5}{ }^{+} \\
\text {abundance }\end{array}$ & $\begin{array}{c}\mathrm{b}_{4}{ }^{+} \\
\text {abundance }\end{array}$ & $\begin{array}{c}\mathrm{b}_{3}^{+} \\
\text {abundance }\end{array}$ & $\begin{array}{c}\mathrm{b}_{2}{ }^{+} \\
\text {abundance }\end{array}$ \\
\hline \hline 25 & $28 \pm 1$ & $18.3 \pm 0.8$ & $12 \pm 4$ & $3.8 \pm 0.8$ & $0 \pm 0$ & $0 \pm 0$ & $0 \pm 0$ & $0 \pm 0$ \\
30 & $63 \pm 1$ & $31.6 \pm 0.8$ & $\mathbf{1 5} \pm \mathbf{2}^{*}$ & $\mathbf{2 8} \pm \mathbf{2}$ & $\mathbf{1 . 6} \mathbf{0 . 5}$ & $\mathbf{2 . 5} \pm \mathbf{0 . 6}$ & $0 \pm 0$ & $0 \pm 0$ \\
40 & $88 \pm 2$ & $34 \pm 1$ & $2.3 \pm 6$ & $25 \pm 2$ & $0.4 \pm 0.3$ & $1.9 \pm 0.5$ & $0.8 \pm 0.8$ & $0 \pm 0$ \\
45 & $95 \pm 2$ & $33.9 \pm 0.9$ & $1.5 \pm 6$ & $25 \pm 1$ & $0 \pm 0$ & $1.8 \pm 0.5$ & $0.7 \pm 0.7$ & $0 \pm 0$ \\
50 & $99 \pm 2$ & $32.3 \pm 0.9$ & $0.3 \pm 0.2$ & $17.4 \pm 0.9$ & $0 \pm 0$ & $0.7 \pm 0.3$ & $0 \pm 0$ & $0.3 \pm 0.2$ \\
\hline
\end{tabular}

*The largest abundance observed for each ion is shown in bold.

the hole in the ring electrode as diameters of a circular beam spot, respectively, the average flux of the focused laser per square millimeter is $6 \pm 1$ times greater than the average flux of the unfocused laser. Only the high-probability area of the ion cloud is irradiated by the focused laser spot instead of a larger area through the trapping volume.

\section{IRMPD Efficiency}

Increased laser flux on the ion cloud produces greater fragmentation efficiency and increases the dependence of fragmentation efficiency on ion cloud size. IRMPD of ions with the laser unfocused and focused was compared at a helium bath gas pressure of $3.3 \times 10^{-4}$ Torr (Figure 2), reduced from $1.0 \times 10^{-3}$ Torr to decrease collisional cooling. By using the focused laser, 7 to 12 times less irradiation time was required to achieve $100 \%$ fragmentation efficiency for respective parent ions compared to IRMPD with the unfocused laser. Fragmentation efficiency at a given irradiation time was also observed to increase with ion mass. Two factors explain the IRMPD mass dependence. Larger peptide ions with more residues have more IR-active groups, increasing absorption and dissociation despite having more degrees of freedom. Larger $m / z$ ions also remain closer to the center of the trap and the most intense part of the laser. The focused laser increases the importance of the second factor because of the greater laser flux at the center of the trap.

IRMPD of ions with the laser unfocused and focused was also compared at a helium bath gas pressure of $1.0 \times 10^{-3}$ Torr (Figure 3). Irradiation times for equivalent fragmentation efficiencies were increased compared to IRMPD at $3.3 \times 10^{-4}$ Torr because of the increased collisional cooling. No more than 3\% fragmentation efficiency could be achieved for any ion after $300 \mathrm{~ms}$ irradiation with the unfocused laser. Less than $30 \mathrm{~ms}$ was required to achieve $100 \%$ fragmentation efficiency for all parent ions with the focused laser. This is 3 to 10 times less irradiation time for respective parent ions compared to IRMPD at $3.3 \times 10^{-4}$ Torr with the unfocused laser. Irradiation time was not as short as IRMPD with a focused laser at $3.3 \times 10^{-4}$ Torr, and smaller ions required a greater relative increase in irradiation time at $1.0 \times 10^{-3}$ Torr. The rate of collisional cooling decreases with increasing mass [23], and the dissociation of larger ions is decreased less by collisional cooling at $1.0 \times 10^{-3}$ Torr. Larger ions also have greater IR activity and are closer to the center of the laser.

A bath gas at $1.0 \times 10^{-3}$ Torr improves detection over lower pressures, and efficient IRMPD at $1.0 \times 10^{-3}$ Torr with the focused laser allows product ions to be observed in greater abundance. Table 1 shows efficiency values and product ion abundances from IRMPD of [ALILTLVS $+\mathrm{H}]^{+}$at $3.3 \times 10^{-4}$ Torr with the unfocused laser. As fragmentation efficiency of the parent ion increased, product ion abundances grew to a maximum and then decreased due to sequential dissociation. MS/MS efficiency did not increase above 34\% with increasing fragmentation efficiency because some product ions were sequentially dissociated to below the LMCO even at a $q_{z}$ of 0.10 . Product ion abundances from IRMPD of [ALILTLVS $+\mathrm{H}^{+}$at $1.0 \times 10^{-3}$ Torr with the focused laser are shown in Table 2 at similar fragmentation efficiencies to Table 1. Absolute product ion abundances were increased four to 40 times with IRMPD at the higher bath gas pressure and there was no change in the background noise. Several product

Table 2. Product ion abundances from IRMPD of [ALILTLVS $+\mathrm{H}]^{+}$with the laser focused at helium bath gas pressure of $1.0 \times$ $10^{-3}$ Torr

\begin{tabular}{|c|c|c|c|c|c|c|c|c|}
\hline $\begin{array}{l}\text { Irradiation } \\
\text { time (ms) }\end{array}$ & $\begin{array}{c}\% \text { Fragmentation } \\
\text { efficiency }\end{array}$ & $\begin{array}{l}\% \mathrm{MS} / \mathrm{MS} \\
\text { efficiency }\end{array}$ & $\begin{array}{c}\mathrm{b}_{7}^{+} \\
\text {abundance }\end{array}$ & $\begin{array}{l}{\left[\mathrm{b}_{6}-\mathrm{H}_{2} \mathrm{O}\right]^{+}} \\
\text {abundance }\end{array}$ & $\begin{array}{c}\mathrm{b}_{5}^{+} \\
\text {abundance }\end{array}$ & $\begin{array}{c}\mathrm{b}_{4}^{+} \\
\text {abundance }\end{array}$ & $\begin{array}{c}\mathrm{b}_{3}^{+} \\
\text {abundance }\end{array}$ & $\begin{array}{l}\quad \mathrm{b}_{2}^{+} \\
\text {abundance }\end{array}$ \\
\hline 3 & $22.3 \pm 0.7$ & 19.6 & $520 \pm 40$ & $23 \pm 3$ & $7 \pm 1$ & $1.5 \pm 0.5$ & $0.3 \pm 0.3$ & $0 \pm 0$ \\
\hline 4 & $65 \pm 2$ & $38.6 \pm 0.7$ & $570 \pm 30^{*}$ & $124 \pm 9 *$ & $29 \pm 2^{*}$ & $19 \pm 2^{*}$ & $7 \pm 2$ & $0.3 \pm 0.2$ \\
\hline 6 & $87 \pm 2$ & $42.6 \pm 0.8$ & $280 \pm 20$ & $88 \pm 6$ & $23 \pm 2$ & $15 \pm 2$ & $26 \pm 3^{*}$ & $8 \pm 1$ \\
\hline 8 & $96 \pm 2$ & $32.9 \pm 0.4$ & $55 \pm 5$ & $65 \pm 5$ & $7.8 \pm 9$ & $3.5 \pm 0.8$ & $22 \pm 2$ & $14 \pm 2^{*}$ \\
\hline 9 & $100 \pm 2$ & $22.4 \pm 0.3$ & $3 \pm 1$ & $25 \pm 3$ & $1.2 \pm 0.8$ & $0.6 \pm 0.4$ & $9 \pm 2$ & $13 \pm 3$ \\
\hline
\end{tabular}

*The largest abundance observed for each ion is shown in bold. 
ions, such as $b_{3}{ }^{+}$and $b_{2}{ }^{+}$, were only reliably observed at $1.0 \times 10^{-3}$ Torr. MS/MS efficiency increased to a maximum of $43 \%$ and was lower at $100 \%$ fragmentation efficiency compared with MS/MS efficiency reported in Table 1. Sequential dissociation of product ions increases from the high flux of the focused laser, and more product ions are formed below the LMCO and not observed, decreasing MS/MS efficiency. Sequential dissociation is further increased for the reduced ion cloud sizes at $1.0 \times 10^{-3}$ Torr, but the greater sensitivity at higher pressure increases observed product ion abundances despite more extensive sequential dissociation.

\section{Conclusions}

Sensitivity is increased and experiment time is decreased by performing IRMPD with a focused laser at $1.0 \times 10^{-3}$ Torr. No modification to the standard IRMPD experiment is required, and the only instrumental modification consists of the simple addition of a lens to the laser optics. Higher sensitivity at $1.0 \times 10^{-3}$ Torr allows product ions to be observed in greater abundance compared to the reduced pressures usually employed for IRMPD. Irradiation time is greatly reduced by focusing the laser beam to a spot similar to the axial amplitudes of trapped ion clouds at a $\mathrm{q}_{z}$ of 0.10 . The laser flux on the ion cloud is increased six times by focusing laser power on the smaller area. IRMPD with the focused laser can dissociate larger peptide ions that have greater overlap with the high-flux laser and more IR-active groups. IRMPD of less easily dissociated ions might now be possible on a timescale no greater than previous experiments with an unfocused laser, such as $<100$ Da volatile organic compounds with few vibrational degrees of freedom, or $>m / z 2000$ nanoparticles with many degrees of freedom and few IR-active groups.

\section{Acknowledgments}

The authors acknowledge support for this work by NSF grant CHE-0431825.

\section{References}

1. Stephenson, J. L.; Booth, M. M.; Shalosky, J. A.; Eyler, J. R.; Yost, R. A. Infrared Multiple Photon Dissociation in the Quadrupole Ion Trap Via a Multipass Optical Arrangement. J. Am. Soc. Mass Spectrom. 1994, 5, 886-893.
2. Colorado, A.; Shen, J. X.; Vartanian, V. H.; Brodbelt, J. Use of Infrared Multiphoton Photodissociation with Swift for Electrospray Ionization and Laser Desorption Applications in a Quadrupole Ion Trap Mass Spectrometer. Anal. Chem. 1996, 68, 4033-4043.

3. Goolsby, B. J.; Brodbelt, J. S. Characterization of B-Lactams by Photodissociation and Collision-Activated Dissociation in a Quadrupole Ion Trap. J. Mass Spectrom. 1998, 33, 705-712.

4. Payne, A. H.; Glish, G. L. Thermally Assisted Infrared Multiphoton Photodissociation in a Quadrupole Ion Trap. Anal. Chem. 2001, 73, 3542-3548.

5. Shen, J.; Brodbelt, J. S. Characterization of Ionophore-Metal Complexes by Infrared Multiphoton Photodissociation and Collision-Activated Dissociation in a Quadrupole Ion Trap Mass Spectrometer. Analyst 2000, 125, 641-650.

6. Crowe, M. C.; Brodbelt, J. S.; Goolsby, B. J.; Hergenrother, P. Characterization of Erythromycin Analogs by Collisional Activated Dissociation and Infrared Multiphoton Dissociation in a Quadrupole Ion Trap. J. Am. Soc. Mass Spectrom. 2002, 13, 630-649.

7. Keller, K. M.; Brodbelt, J. S. Collisionally Activated Dissociation and Infrared Multiphoton Dissociation of Oligonucleotides in a Quadrupole Ion Trap. Anal. Biochem. 2004, 326, 200-210.

8. Little, D. P.; Speir, J. P.; Senko, M. W.; O'Connor, P. B.; McLafferty, F. W. Infrared Multiphoton Dissociation of Large Multiply Charged Ions for Biomolecule Sequencing. Anal. Chem. 1994, 66, 2809-2815.

9. Little, D. P.; Aaserud, D. J.; Valaskovic, G. A.; McLafferty, F. W. Sequence Information from 42-108-Mer DNAs (complete for a 50-Mer) by Tandem Mass Spectrometry. J. Am. Soc. Mass Spectrom. 1996, 118, 9352-9359.

10. Xie, Y.; Lebrilla, C. B. Infrared Mulitphoton Dissociation of Alkali Metal-Coordinated Oligosaccharides. Anal. Chem. 2003, 75, 1590-1598.

11. Sleno, L.; Volmer, D. A. Ion Activation Methods for Tandem Mass Spectrometry. J. Mass Spectrom. 2004, 39, 1091-1112.

12. Stafford, G. C. J.; Kelley, P. E.; Syka, J. E. P.; Reynolds, W. E.; Todd, J. F. J. Recent Improvements in and Analytical Applications of Advanced Ion Trap Technology. Int. J. Mass Spectrom. Ion Processes 1984, 60, 85-98.

13. Black, D. M.; Payne, A. H.; Glish, G. L. Determination of Cooling Rates in a Quadrupole Ion Trap. J. Am. Soc. Mass Spectrom. 2006, 17, 932-938.

14. Hashimoto, Y.; Hasegawa, H.; Yoshinari, K.; Waki, I. CollisionActivated Infrared Multiphoton Dissociation in a Quadrupole Ion Trap Mass Spectrometer. Anal. Chem. 2003, 75, 420-425.

15. Boué, S. M.; Stephenson, J. L.; Yost, R. A. Pulsed Helium Introduction into a Quadrupole Ion Trap for Reduced Collisional Quenching During Infrared Multiphoton Dissociation of Electrosprayed Ions. Rapid Commun. Mass Spectrom. 2000, 14, 1391-1397.

16. Pikulski, M.; Wilson, J. J.; Aguilar, A.; Brodbelt, J. S. Amplification of Infrared Multiphoton Dissociation Efficiency in a Quadruple Ion Trap Using Ir-Active Ligands. Anal. Chem. 2006, 78, 8512-8517.

17. Pikulski, M.; Hargrove, A.; Shabbir, S. H.; Anslyn, E. V.; Brodbelt, J. S Sequencing and Characterization of Oligosaccharides Using Infrared Multiphoton Dissociation and Boronic Acid Derivatization in a Quadrupole Ion Trap. J. Am. Soc. Mass Spectrom. 2007, 18, 2094-2106.

18. Laskin, J.; Futrell, J. H. Activation of Large Ions in FT-ICR Mass Spectrometry. Mass Spectrom. Rev. 2005, 24, 135-167.

19. Yates, N.; Yost, R. 1992. ICMS Ion Trap Software. Release 2.20 unpublished, University of Florida, Gainesville, FL.

20. Hemberger, P. H.; Nogar, N. S.; Williams, J. D.; Cooks, R. G.; Syka, J. E. P. Laser Photodissociation Probe for Ion Tomography Studies in a Quadrupole Ion-Trap Mass Spectrometer. Chem. Phys. Lett. 1992, 191, 405-410.

21. Williams, J. D.; Cooks, R. G.; Syka, J. E. P.; Hemberger, P. H.; Nogar, N. S. Determination of Positions, Velocities, and Kinetic Energies of Resonantly Excited Ions in the Quadrupole Ion Trap Mass Spectrometer by Laser Photodissociation. J. Am. Soc. Mass Spectrom. 1993, 4, 792-797.

22. Remes, P. M.; Glish, G. L. Mapping the Distribution of Ion Positions as a Function of Quadrupole Ion Trap Mass Spectrometer Operating Parameters to Optimize Infrared Multiphoton Dissociation. J. Phys. Chem. A 2009, in press.

23. Goeringer, D. E.; McLuckey, S. A. Relaxation of Internally Excited High-Mass Ions Simulated under Typical Quadrupole Ion Trap Storage Conditions. Int. J. Mass Spectrom. 1998, 177, 163-174. 